\title{
Incidence and impact of hyperviscosity on sperm parameters of Malawian men seeking assisted reproduction
}

\author{
Fanuel Lampiao, Joseph Chisaka
}

Department of Biomedical Sciences, Division of Physiology, College of Medicine, P.O. Box 360, Blantyre 3, Malawi.

\begin{abstract}
Background: Seminal hyperviscosity has been shown to be associated with male infertility. The aim of this study was to assess the prevalence of hyperviscosity in semen of Malawian males seeking infertility treatment.

Methods: A total of 120 men visiting our laboratory for fertility assessment donated semen samples. The semen samples were assessed for hyperviscosity, volume, concentration, total motility, progressive motility, viability, and morphology.

Results: Out of the 120 samples analyzed, 34 samples were hyperviscous representing $28.3 \%$. No significant statistical difference in semen volume between samples with normal viscosity compared to those with hyperviscosity ( $\mathrm{p}>0.05)$. Sperm concentration, progressive motility, total motility, viability, and normal morphology were significantly higher in the normal viscosity group when compared to the abnormal viscocity group $(\mathrm{p}<0.05)$.

Conclusion: Hyperviscosity affects a significant number of men in Malawi and may be the cause of decreased fertility as it was associated with poor sperm concentration, total motility, progressive motility, viability, and morphology.

Keywords: Viscosity, sperm motility, accessory sex glands, infertility, semen, semen analysis, spermatozoa.

DOI: https://dx.doi.org/10.4314/ahs.v20i1.3

Cite as: Lampiao F, Chisaka J. Incidence and impact of hyperviscosity on sperm parameters of Malawian men seeking assisted reproduction. Afri Health Sci. 2020;20(1):1-3. bttps:/ / dx.doi.org/10.4314/ahs.v20i1.3
\end{abstract}

\section{Introduction}

The basic semen analysis plays a very important role in the diagnosis of male infertility ${ }^{1}$. It is a valuable diagnostic tool in assessing disorders affecting sperm production and the secretory pattern of the male accessory sex glands. This information can be used to determine the fertility status of the male.

Male factor infertility has been reported to contribute $30-40 \%$ of sub-fertile cases in couples ${ }^{2}$. There are many factors that contribute to male factor infertility such as reactive oxygen species, leukocytospermia, varicocele, and DNA damage of spermatozoa ${ }^{3}$. Hyperviscosity of semen is another condition that has been shown to contribute to

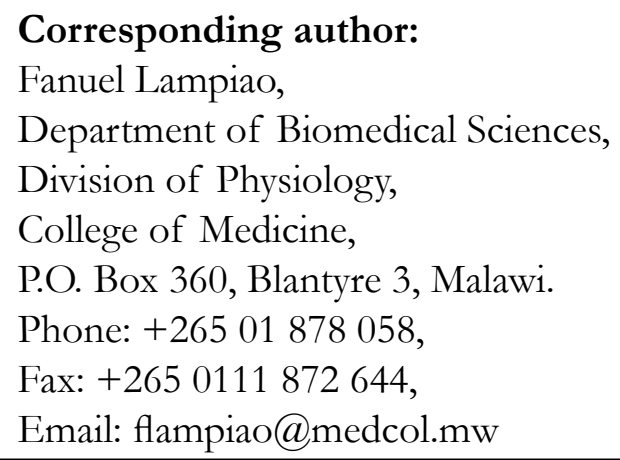

male factor infertility because it leads to changes in the chemical and physical characteristics of semen ${ }^{4}$. Viscosity measures the seminal fluid's resistance to flow. Hyperviscosity may interfere with determination of sperm motility, and concentration.

Usually normal semen sample shows liquefaction from 15-60 minutes of ejaculation ${ }^{5}$. A liquefied semen sample has low viscosity, but if intensive viscous properties remain over time after liquefaction, it is regarded as hyperviscous. This study was aimed at assessing the prevalence of hyperviscous semen samples from sub-fertile males seeking assisted reproduction and how hyperviscosity may affect sperm parameters.

\section{Materials and methods}

\section{Study area, setting and subjects}

The study was carried out at the College of Medicine Andrology Laboratory in Blantyre, Malawi. The study sample consisted of 120 male partners of infertile couples who had infertility for more than one year and who sought their first infertility evaluation. Men with a medical history of inflammation, and sexually transmitted infections were excluded from the study. All men enrolled 
in this study gave a written consent after the procedures had been described to them.

\section{Semen collection and analysis}

Semen samples were obtained by masturbation in a room next to the laboratory after 3-5 days of sexual abstinence. Semen assessment was performed after 60 minutes of collection as described by the World Health Organization $^{5}$. Seminal volume was measured in a graduated pipette accurate to within $0.1 \mathrm{~mL}$. The viscosity of a semen sample was estimated by aspirating it into a plastic pipette ( $\pm 1.5 \mathrm{~mm}$ diameter) and allowing the semen to drop by gravity and observing the length of any thread formed. A sample was considered normal if it leaves the pipette in small discrete drops. A sample was regarded as hyperviscous when the drops formed a thread of more than $2 \mathrm{~cm}$ long. Sperm concentration, total motility and progressive motility were assessed by direct observation under a microscope $(\times 400)$. To assess viability drops were placed on glass slides and mixed with eosin-nigrosin dye. Smears were made on the slides and left to air-dry. Spermatozoa with impaired metabolism or damaged membranes allowed te eosin-nigrosin dye to enter the cell and stain the DNA. The slides were observed under a light microscope and at least 200 cells were evaluated on each slide. For morphology assessment, smears were made on clean slides and air dried after which they were stained with hemacolor (Merck, Darmstadt, Germany). Morphology was analyzed by oil immersion light microscopy according to the Tygerberg strict criteria.

\section{Statistical analysis}

Data are expressed as mean \pm SD and the level of significance for comparison set at $\mathrm{P}<0.05$. Comparisons between the two groups were made using the Chi-square test for categorized independent variables and the t-test for continuous independent variables.

\section{Results}

Our results showed that out of the 120 samples analyzed, 34 samples were hyperviscous representing $28.3 \%$. Table 1 shows that there was no statistically significant difference in semen volume between samples with normal viscosity compared to those with hyperviscosity $(3.8 \pm 0.84 \mathrm{~mL}$ vs $3.6 \pm 0.55 \mathrm{~mL}, \mathrm{p}>0.05)$. Sperm concentration, progressive motility, total motility, viability, and normal morphology were significantly higher in the normal viscosity group when compared to the abnormal viscocity group $(35.3 \pm 9.43 \% \times 106 / \mathrm{mL}$ vs $19.4 \pm 8.85 \%$ $\mathrm{x} 106 / \mathrm{mL} ; 31.7 \pm 8.56 \%$ vs $22.2 \pm 7.68 \% ; 63 \pm 11.40 \%$ vs $52.8 \pm 8.44 \% ; 78 \pm 8.45 \%$ vs $57 \pm 11.35 \% ; 19.2 \pm 5.64$ vs $12.8 \pm 6.93, \mathrm{p}<0.05)$ respectively.

Table 1: Semen parameters of hyperviscous and normal samples

\begin{tabular}{lcc}
\hline $\begin{array}{l}\text { Samples with normal } \\
\text { viscosity }(\mathrm{n}=86)\end{array}$ & $\begin{array}{l}\text { Samples with abnormal } \\
\text { viscosity }(\mathrm{n}=34)\end{array}$ \\
Variables & $3.8 \pm 0.84$ & $3.6 \pm 0.55$ \\
\hline Semen volume $(\mathrm{mL})$ & $35.3 \pm 9.43$ & $19.4 \pm 8.85^{*}$ \\
Sperm concentration $(10 \% \mathrm{~mL})$ & $22.2 \pm 7.68^{*}$ \\
Progressive motility $(\%)$ & $31.7 \pm 8.56$ & $52.8 \pm 8.44^{*}$ \\
Total motility (\%) & $63 \pm 11.40$ & $57 \pm 11.35^{*}$ \\
Viability (\%) & $78 \pm 8.45$ & $12.8 \pm 6.93 *$ \\
Normal Morphology $(\%)$ & $19.2 \pm 5.64$ & \\
& & \\
\hline
\end{tabular}

$* \mathrm{P}<0.05$ 


\section{Discussion}

In this study we found that $28 \%$ of the male partners seeking fertility testing had semen which was hyperviscous. As far as we are concerned, this is the first study to report the prevalence of semen hyperviscosity in Malawian men. This finding is similar to those reported in previous studies that seminal hyperviscosity occurs in $12 \%-29 \%$ of ejaculates ${ }^{1,6}$. Our findings have also shown that hyperviscosity is assocated with low sperm concentration, low sperm total motility, progressive motility, low sperm viability, and low normal morphlogy which is consistent with previous studies ${ }^{4,7}$. The semen samples used in this study were from men who had tried to have a children for more than one year. We speculate that hyperviscosity could have been one of the contributing factors to their infertility.

In this study we used the WHO method for the assessment of hyperviscosity which is simple and can be used in any laboratory. However, the method does not allow defining the exact composition of the semen. Biochemical characterization of seminal fluid may help to elucidate the mechanism and causes of hyperviscosity. Studies have reported that semen coagulation and liquefaction depends on the proteins that come from the secretions of the accessory glands such as the seminal vescicles and prostate $^{8,9}$. If there is a dysfunction of these glands, it will lead to hyperviscosity.

Hyperviscosity has been linked to infertility as it negatively affects sperm and semen parameters ${ }^{3}$. It physically affects the movement of sperm. Hyperviscosity has been reported to affect kinetic parameters such as motility, velocity, and linear direction of sperm ${ }^{10}$. This could trap sperm in the female reproductive tract and inhibit normal movement.

\section{Conclusion}

We established the prevalence rate of semen hyperviscosity in men seeking fertility treatment in Malawi. The hyperviscosity was associated with poor sperm concetra- tion, total motility and progressive motility. Hyperviscosity affects a significant number of men in Malawi and may be the cause of decreased fertility.

\section{Conflict of interest}

The authors declare no conflict of interest.

\section{References}

1. Andrade-Rocha FT. Physical analysis of ejaculate to evaluate the secretory activity of the seminal vesicles and prostate. Clin Chem Lab Med. 2005;43: 1203-1210.

2. Thonneau P, Marchand S, Tallec A, Ferial ML, Ducot B, Lansac J, Lopes P, Tabaste JM, Spira A. Incidence and main causes of infertility in a resident population $(1,850,000)$ of three French regions (1988-1989). Hum Reprod. 1991;6: 811-816.

3. Du Plessis SS, Gokul S, Agarwal A. Semen hyperviscosity: causes, consequences, and cures. Frontiers in Bioscience, Elite 2013; 5:224-231.

4. Gonzales GF, Kortebani G, Mazzolli AB. Hyperviscisity and hypofunction of the seminal vesicles. Arch Androl. 1993;30: 63-68.

5. WHO. WHO Laboratory Manual for the Examination and Processing of Human Semen. 2010; 5th Edition. Geneva.

6. Wilson VB, Bunge RG. Infertility and semen non-liquefaction. J Urol. 1975;113: 509-510.

7. Elzanaty S, Malm J, Giwercman A. Visco-elasticity of seminal fluid in relation to the epididymal and accessory sex gland function and its impact on sperm motility. Int $J$ Androl. 2004; 27:94-100.

8. Bjartell A, Malm J, Moller C, Gunnarsson M, Lundwall A, Lilja H. Distribution and tissue expression of semenogelin I and II in man as demonstrated by in situ hybridization and immunocytochemistry. J Androl. 1996; 17:17-26. 9. Schill WB. Significance of preteolytic sperm enzymes for the fertility. Hautarzt 1975; 26:514-523.

10. Mendeluk GR, Munuce MJ, Carizza C, Sardi M, Bregni C. Sperm motility and ATP content in seminal hyperviscocity. Arch Androl. 1997; 39:223-227. 